\title{
Lisímetro de pesagem de grande porte. Parte II: Consumo hídrico do coqueiro anão verde irrigado
}

\author{
Inajá F. Sousa ${ }^{1}$, Antenor O. A. N etto ${ }^{1}$, Luiz F. M. S. Campeche ${ }^{2}$, Allan C. Barros ${ }^{3}$, \\ Vicente de P. R. da Silva ${ }^{4} \&$ Pedro V. de Azevedo ${ }^{4}$
}

\begin{abstract}
RESU MO
Este trabalho, como segunda parte de uma pesquisa realizada no Estado de Sergipe, objetiva determinar a evapotranspiração e o coeficiente de cultura na fase de crescimento do coqueiro anão-verde (Cocos nucifera L.), com base em medições lisimétricas e no modelo do balanço de energia, segundo a razão de Bowen. O bteve-se a evapotranspiração de referência pelo méto do de Penman-M onteith, na escala diária em todo o período experimental. O sistema de aquisição de dados foi programado para a automação da coleta de todos os sensores necessários à obtenção dos componentes do balanço de energia. 0 consumo hídrico do coqueiro durante a fase de crescimento é de $1.263,30 \mathrm{~mm}$, com média diária de 3,90 $\mathrm{mm} \mathrm{d}^{-1}$. 0 coeficiente de cultura nessa fase fenológica da palmeira varia entre 0,50 e 1,80, com média de 0,96.
\end{abstract}

Palavras-chave: evapotranspiração, coeficiente de cultura, balanço de energia

\section{Large-scale weighing lysimeter. Part II: W ater requirements of the irrigated dwarf-green coconut}

\begin{abstract}
This paper, as Part II of a research carried out in Sergipe state, aims to determine evapotranspiration and crop coefficient of dwarf-green coconut (Cocos nucifera L.) based on lysimeter measurements and Bow en ratio-energy balance method. The reference evapotranspiration was obtained by the Penman-M onteith approach on daily-scale during the experimental period. The data acquisition system was used to obtain all data from the sensors necessary to determine the energy balance components. The water requirements of coconut palm during the phenological growth stage is $1263.30 \mathrm{~mm}$, with daily average of $3.90 \mathrm{~mm} \mathrm{~d}^{-1}$. The crop coefficient during this phenological growth stage varies between 0.50 and 1.80 , with daily mean of 0.96 .
\end{abstract}

Key words: evapotranspiration, crop coefficient, energy balance

\footnotetext{
${ }^{1}$ DEA/U FS, Cidade U niversitária Prof. José Aloísio de Campos, CEP 49100-000, São Cristóvão, SE. Fone: (79) 2105-6929. E-mail: ifsousa@ufs.br; antenor@ufs.br

${ }^{2}$ Instituto Federal de Educação, Ciência e Tecnologia do Sertão Pernambucano, BR 407, km 8, Jardim São Paulo, CEP 56000-000, Petrolina, PE. E-mail: Ifsmcamp@carpa.ciagri.usp.br

${ }^{3}$ UFAL, BR $101 \mathrm{~N}$ orte, km 97, Tabuleiro do M artins, CEP 57072-970, Maceió, AL. Fone: (82) 3322-2170. E-mail: allan.cunha.barros@gmail.com

${ }_{4}^{4}$ U ACA/U FCG , Av. A prígio Veloso 882, Bodocongó, CEP 58109-970, Campina G rande, PB. Fone (83) 3310-1202. E-mail: vicente@dca.ufcg.edu.br; pvieira@dca.ufcg.edu.br
} 


\section{INTRODUÇÃO}

Esta é a segunda parte da pesquisa sobre a utilização de lisímetro de grande porte na determinação do consumo hídrico do coqueiro anão-verde. Na primeira parte do trabalho são apresentados os detalhes do desenvolvimento e a calibração do lisímetro, enquanto a segunda parte trata da comparação das medições da evapotranspiração realizadas pelo equipamento com os dados do modelo micrometeorológico do balanço de energia baseado na razão de Bowen, além da determinação do coeficiente de cultura do coqueiro.

O coqueiro (Cocos nucifera L.) é uma palmeira essencialmente tropical cultivada, em geral, em condições climáticas favoráveis entre as latitudes $20^{\circ} \mathrm{Ne} 20^{\circ} \mathrm{S}$. O Brasil é líder mundial na produção de coco verde, com área cultivada equivalente a 57 mil hectares distribuídos praticamente em quase todo o território nacional, sobretudo na faixa litorânea (Lins et al., 2003). O Nordeste é uma das regiões produtoras de frutos mais importantes do Brasil, em razão das excelentes condições edafoclimáticas para o cultivo de plantas frutíferas. O cultivo do coqueiro requer clima quente com pouca variação na temperatura do ar e precipitação bem distribuída ao longo do ano (Sousa et al., 2006). Esta palmeira é uma das plantas perenes mais capazes de gerar um sistema autossustentável de exploração, sendo o coco, também, uma importante fonte de proteínas, além de geradora de divisas para a população litorânea dos estados do Nordeste do Brasil, haja vista que ela produz, também, matéria-prima para uma centena de produtos, em mais de 86 países localizados na zona intertropical do globo terrestre.

O coqueiro anão-verde tem grande variedade de utilidades, com significativo destaque no mercado internacional, por fornecer óleo, gorduras, minerais e vitaminas essenciais e fruto fresco (Azevedo et al., 2006); a casca do coco é usada na fabricação de cordas, tapetes, chapéus e encosto de veículos, enquanto o óleo é largamente usado na indústria alimentícia, como óleo de mesa e na produção de margarina, glicerol, cosméticos, detergentes sintéticos, sabão, velas e fluidos para freio de avião. Em função da rentabilidade financeira e do crescente consumo da água de coco nos grandes centros urbanos, é notório o interesse de produtores por essa palmeira. Aliado aos aspectos positivos do mercado, o coqueiro anãoverde, dependendo da tecnologia utilizada, pode florescer com aproximadamente dois anos de idade e atingir, em função dos tratos culturais dispensados, mais de 200 frutos planta ${ }^{-1}$ ano $^{-1}$, o que proporciona maior rapidez no retorno dos investimentos realizados.

As necessidades hídricas do coqueiro dependem de vários fatores, tais como a idade da planta (altura e área foliar), o clima local (radiação solar, temperatura, umidade relativa do ar e velocidade do vento), o teor de umidade do solo, o método de irrigação utilizado e o estado nutricional da planta. Esta palmeira é, em condições irrigadas, uma das maiores consumidoras de água, tornado-se oportuno, então, o conhecimento de suas reais necessidades hídricas, visando maximizar o uso da água (Sousa et al., 2006). A utilização de medições diretas da evapotranspiração dessa palmeira, através de lísimetros ou por modelos micrometeorlógicos, como o balanço de energia baseado na razão de Bowen, constitui-se numa ação imprescindível para o conhecimento das exigências hídricas do coqueiro.

Os lisímetros de pesagem realizam medições diretas das perdas de água da superfície do solo e vegetação (evapotranspiração) para a atmosfera e, portanto, fornecem os dados básicos para validar outros modelos de predição da evapotranspiração (Vaughan et al., 2007). López-Urrea et al. (2006) compararam a evapotranspiração de referência obtida por sete diferentes métodos climáticos com dados de lisímetros de pesagem em Albacete, Espanha, com precisão de $250 \mathrm{~g}$, ou seja, a 0,04 mm de água, e constataram que o método de PenmamMonteith é o mais adequado para se estimar a evapotranspiração em regiões semiáridas quando comparado com medições lisimétricas. Outros estudos realizados em diferentes partes do mundo têm comparado a evapotranspiração de culturas obtidas em lisímetros com dados do balanço de energia baseado na razão de Bowen (Silva et al., 2005; Gavilán \& Berengena, 2007). Este método micrometeorológico tem sido bastante empregado na determinação do consumo hídrico de diferentes frutíferas na região Nordeste do Brasil, tal como para mangueira (Azevedo et al., 2003; Borges et al., 2008; Silva et al., 2009), para a videira (Azevedo et al., 2008) e para o abacaxizeiro (Souza et al., 2008). Por outro lado e não obstante os trabalhos de Azevedo et al. (2006) e de Sousa et al. (2006) sobre a eficiência de uso de água e a evapotranspiração do coqueiro, modelado no balanço hídrico no solo, o estudo do consumo hídrico desta palmeira, determinado seguindo as medições lisimétricas, pode contribuir com seu manejo de irrigação na região Nordeste do Brasil, razão pela qual o presente trabalho objetivou verificar a eficiência do método do balanço de energia baseado na razão de Bowen, na estimativa da evapotranspiração do coqueiro anão-verde quando comparado às medições lisímetricas e determinar o coeficiente de cultura para a fase de crescimento dessa palmeira cultivada na região Nordeste do Brasil.

\section{Material E MÉTOdos}

\section{Local do experimento e tratos culturais}

$\mathrm{O}$ experimento de campo com o coqueiro anão-verde foi conduzido em área de 166 ha, durante os anos de 2005 e 2006. Os detalhes do clima e solo da região onde foi realizada a pesquisa, são apresentados na Parte I deste trabalho e as médias climatológicas das principais variáveis meteorológicas na área de estudo, são exibidas na Tabela 1.

Fez-se, após a calagem da área, a abertura de covas para o plantio com auxílio de uma broca. As proporções de fertilizantes utilizadas na manutenção da fertilidade do solo na área experimental e no interior do lisímetro, além dos detalhes da irrigação no campo experimental, são apresentados na Parte I do trabalho; assim, o plantio das mudas de coqueiro na área experimental e no lisímetro foi realizado nos dias 25 e 26 de maio de 2005; entretanto, o lisímetro começou a ser operado apenas em 1 de setembro de 2005, quase 4 meses; portanto, após o plantio da muda de coqueiro, tempo suficiente para a acomodação e estabilização da umidade do solo e para o enraizamento da palmeira. $\mathrm{O}$ pomar de coqueiro anão-verde foi 
Tabela 1. Dados médios mensais climatológicos da temperatura máxima do $\operatorname{ar}\left(\mathrm{Tx},{ }^{\circ} \mathrm{C}\right)$, temperatura mínima do $\operatorname{ar}\left(\mathrm{Tm},{ }^{\circ} \mathrm{C}\right)$, temperatura média do $\operatorname{ar}\left(\mathrm{T},{ }^{\circ} \mathrm{C}\right)$, preci pitação (Prec, $\mathrm{mm}$ ), umidade relativa (UR, \%), velocidade do vento $\left(V V, \mathrm{~m} \mathrm{~s}^{-1}\right)$, evaporação do tanque "classe A" (EV, mm) e da insolação (Ins, h) na estação do Campus Rural, São Cristovão, SE (latitude: $11^{\circ} 01^{\prime}$; longitude $=370$ 12'; altitude: $20 \mathrm{~m}$ )

\begin{tabular}{lcccccccc}
\hline Meses & Tx & Tm & T & Prec & UR & VV & EV & Ins \\
Jan & 31 & 21 & 25 & 68 & 77 & 1,8 & 112 & 252 \\
Fev & 31 & 21 & 26 & 81 & 75 & 1,6 & 103 & 226 \\
Mar & 31 & 21 & 26 & 106 & 78 & 1,6 & 105 & 226 \\
Abr & 30 & 21 & 25 & 212 & 80 & 1,4 & 91 & 218 \\
Mai & 28 & 20 & 24 & 250 & 80 & 1,1 & 80 & 190 \\
Jun & 27 & 20 & 23 & 218 & 80 & 1,4 & 79 & 186 \\
Jul & 26 & 19 & 22 & 211 & 80 & 1,3 & 79 & 180 \\
Ago & 26 & 18 & 22 & 128 & 77 & 1,5 & 92 & 200 \\
Set & 28 & 19 & 23 & 83 & 77 & 1,8 & 97 & 216 \\
Out & 29 & 20 & 25 & 62 & 77 & 1,7 & 104 & 246 \\
Nov & 31 & 21 & 25 & 48 & 78 & 1,8 & 100 & 250 \\
Dez & 31 & 21 & 26 & 44 & 76 & 1,9 & 105 & 270 \\
\hline Média & 29 & 20 & 24 & - & 78 & 1,6 & 95,6 & 221,7 \\
\hline Total & - & - & - & $1.511,7$ & - & - & 1.147 & 2.660 \\
\hline
\end{tabular}

cultivado no espaçamento triangular de $8,5 \times 8,5 \times 8,5 \mathrm{~m}$, totalizando 172 plantas por hectare.

\section{Evapotranspiração da cultura}

Para determinação da evapotranspiração da fase de crescimento do coqueiro anão-verde, foram utilizados o lisímetro de pesagem de grande porte, conforme descrito na Parte I deste trabalho, e o método do balanço de energia, baseado na razão de Bowen. Este método segue o princípio de conservação de energia, cujos componentes foram obtidos de acordo com a seguinte expressão (Silva et al., 2007):

$$
\mathrm{R}_{\mathrm{n}}+\mathrm{LE}+\mathrm{H}+\mathrm{G}=0
$$

em que $R_{n}$ é o saldo de radiação, $L E$ e $H$ são os fluxos verticais de calor latente e sensível, respectivamente; $\mathrm{G}$, o fluxo de calor no solo. Todos os termos da Eq. 1 são expressos em W m${ }^{-2}$. O balanço de energia foi efetuado sobre o dossel do pomar de coqueiros, para um volume de controle compreendido entre a profundidade de $0,02 \mathrm{~m}$ do solo e a altura do psicrômetro mais elevado, que foram ajustados de acordo com o crescimento da planta. Os fluxos verticais que chegam foram considerados positivos e os que saem do volume de controle foram considerados negativos (Borges et al., 2008).

O termo $\beta$, referente à razão de Bowen, corresponde à fração de energia entre os fluxos de calor sensível e calor latente, dado pela Eq. 2:

$$
\beta=\frac{\mathrm{H}}{\mathrm{LE}}
$$

A Eq. 2 pode, ainda, ser escrita na forma:

$$
\beta=\frac{\mathrm{Pc}_{\mathrm{P}}}{\mathrm{L} \in}\left(\frac{\mathrm{K}_{\mathrm{h}}}{\mathrm{K}_{\mathrm{w}}}\right) \frac{\partial \mathrm{T} / \partial \mathrm{Z}}{\partial \mathrm{e} / \partial \mathrm{Z}}=\gamma \frac{\mathrm{K}_{\mathrm{h}}}{\mathrm{K}_{\mathrm{w}}} \frac{\Delta \mathrm{T}}{\Delta \mathrm{e}}
$$

em que $\gamma$ é o fator psicrométrico $\left(\mathrm{kPa}^{\circ} \mathrm{C}^{-1}\right), \Delta \mathrm{T}$ é o gradiente da temperatura do $\operatorname{ar}\left({ }^{\circ} \mathrm{C}\right), \Delta e$ é o gradiente da pressão parcial do vapor d'água, $\mathrm{K}_{\mathrm{h}} \mathrm{e} \mathrm{K}_{\mathrm{w}}$ são os coeficientes de difusão turbulenta de calor sensível e de vapor d'água, respectivamente.

Na ausência de advecção de calor sensível, regional ou local e em condição de instabilidade atmosférica, $\mathrm{K}_{\mathrm{h}}=\mathrm{K}_{\mathrm{w}}$; o fluxo de calor latente foi obtido substituindo-se a razão de Bowen na Eq. 1; portanto:

$$
\mathrm{LE}=\frac{\mathrm{R}_{\mathrm{n}}+\mathrm{G}}{1+\beta}
$$

A pressão parcial de vapor d'água em dois níveis acima da copa da planta foi calculada pela equação de Ferrel, expressa da seguinte forma:

$$
e\left(t_{a}\right)=e_{s}\left(t_{u}\right)-0,00066\left(1+0,00115 t_{u}\right)\left(t_{a}-t_{u}\right) \bar{P}
$$

em que $t_{u}$ e $t_{a}$ são as temperatura dos bulbos úmido e seco, respectivamente, expressas em ${ }^{\circ} \mathrm{C}$; $\overline{\mathrm{P}}$, a pressão atmosférica média $(\mathrm{hPa})$ e $\mathrm{e}_{\mathrm{s}}\left(\mathrm{t}_{\mathrm{u}}\right)$ é a pressão de saturação do vapor d'água $(\mathrm{hPa})$, calculada pela equação de Tetens, como:

$$
e_{s}\left(t_{u}\right)=6,1078 \exp \left(\frac{17,269 t_{u}}{237,3+t_{u}}\right)
$$

Os valores de $\beta$ passaram pela análise de consistência de dados conforme estabelecido por Unland et al. (1996). Tal procedimento ocorreu quando $\beta$ se aproximou de -1 , o que resultaria em valores incorretos de LE.

\section{Evapotranspiração de referência}

A evapotranspiração de referência foi calculada pelo método de Penman-Monteith, considerando-se a resistência estomática de $70 \mathrm{~s} \mathrm{~m}^{-1}$, albedo de $23 \%$ e a altura da cultura hipotética fixada em 0,12 m (Allen et al., 1998):

$$
\mathrm{ET}_{\mathrm{o}}=\frac{0,408 \Delta\left(\mathrm{R}_{\mathrm{n}}-\mathrm{G}\right)+\gamma\left(\frac{900 \mathrm{U}_{2}}{\mathrm{~T}+273}\right)\left(\mathrm{e}_{\mathrm{s}}-\mathrm{e}_{\mathrm{a}}\right)}{\Delta+\gamma\left(1+0,34 \mathrm{U}_{2}\right)}
$$

em que $\mathrm{ET}_{\mathrm{o}}$ é a evapotranspiração de referência $\left(\mathrm{mm} \mathrm{d}^{-1}\right), \mathrm{R}_{\mathrm{n}}$ (saldo de radiação) e $\mathrm{G}$ (densidade do fluxo de calor no solo) expressos em $\mathrm{MJ} \mathrm{m}^{-2} \mathrm{~d}^{-1} ; \Delta$ é a declinação da curva de saturação do vapor da água $\left(\mathrm{kPa}^{\circ} \mathrm{C}^{-1}\right)$ e $\mathrm{U}_{2}$ é a velocidade do vento (média diária) a $2 \mathrm{~m}$ acima da superfície do solo $\left(\mathrm{m} \mathrm{s}^{-1}\right)$, T é a temperatura do $\operatorname{ar}\left({ }^{\circ} \mathrm{C}\right), e_{\mathrm{s}}$ é a pressão de saturação do vapor de água $(\mathrm{kPa})$, $\mathrm{e}_{\mathrm{a}}$ é a pressão real do vapor $(\mathrm{kPa})$ e $\gamma$ é o fator psicrométrico $\left(\mathrm{MJ} \mathrm{kg}^{-1}\right)$. Os valores diários da evapotranspiração de referência foram obtidos com base no software SEVAP(Silva et al., 2005), utilizando-se os dados climáticos locais. 
O sistema de aquisição de dados (data logger), utilizado para registrar as variações da célula de carga no lisímetro, também foi programado para a automação da coleta de todos os sensores necessários à obtenção dos componentes do balanço de energia. O data logger foi programado para fazer leituras a cada segundo e extrair médias a cada 30 minutos.

O fornecimento de energia foi feito através de um painel solar, instalado com a finalidade de manter a carga da bateria que fornecia energia ao coletor de dados. Para estabilização da qualidade de energia foi acoplado, então, à bateria e ao data logger, um estabilizador e conversor de voltagem. As variáveis foram medidas através de uma torre micrometeorológica, instalada sobre uma estrutura de andaimes, para facilitar o deslocamento vertical da torre, ao longo das fases de desenvolvimento do coqueiro, cuja finalidade era acoplar os sensores do saldo de radiação, psicrômetros de ventilação forçada, dois fluxímetros instalados a $0,02 \mathrm{~m}$ de profundidade, painel solar e o data logger.

\section{Coeficiente de cultura}

O coeficiente de cultura $(\mathrm{Kc})$, para a fase de crescimento do coqueiro anão-verde foi determinado pela relação entre a evapotranspiração da cultura (ETc), obtida com base em medições lisimétricas e a evapotranspiração de referência (ETo), obtida pelo método de Penman-Monteith (Allen et al., 1998):

$$
\mathrm{K}_{\mathrm{c}}=\frac{\mathrm{ET}_{\mathrm{c}}}{\mathrm{ET}_{\mathrm{o}}}
$$

\section{Análise estatística}

A precisão do modelo de estimativa da evapotranspiração da cultura pelo método da razão de Bowen $\left(\mathrm{ET}_{\text {Bowen }}\right.$ ) foi avaliada com base no coeficiente de correlação (r), no índice de concordância (d) (Willmott, 1982) e no coeficiente de confiança ou desempenho (c), o qual é o produto entre r e d.

$$
\mathrm{d}=1-\left[\frac{\sum_{\mathrm{i}}^{\mathrm{n}}\left(\mathrm{P}_{\mathrm{i}}-\mathrm{O}_{\mathrm{i}}\right)^{2}}{\sum_{1}^{\mathrm{n}}\left(\left|\mathrm{P}_{\mathrm{i}}-\mathrm{O}_{\text {med }}\right|+\mid \mathrm{O}_{\mathrm{i}}-\mathrm{O}_{\text {med }}\right)^{2}}\right]
$$

O índice $\mathrm{c}=0$ indica confiança nula $\mathrm{e}$ o índice $\mathrm{c}=1$ significa confiança perfeita. Também se obteve o erro quadrático médio (EQM) pela seguinte equação:

$$
\mathrm{EQM}=\left[\frac{1}{\mathrm{n}} \sum_{1}^{\mathrm{n}}\left(\mathrm{P}_{\mathrm{i}}-\mathrm{O}_{\mathrm{i}}\right)^{2}\right]^{0,5}
$$

em que $\mathrm{n}$ é o número de dados, $\mathrm{P}_{\mathrm{i}}$ é a $\mathrm{ET}_{\text {Bowen }}$ estimada pelo modelo a ser avaliado $\left(\mathrm{mm} \mathrm{d}^{-1}\right), \mathrm{O}_{i}$ é a evapotranspiração obtida pelo lisímetro $\left(\mathrm{ET}_{\text {lisímetro }}\right)$, em mm d $\mathrm{eO}_{\text {med }}$ é a média de $\mathrm{ET}_{\text {lisímetro }}$ $\left(\mathrm{mm} \mathrm{d}^{-1}\right)$. O índice de confiança ou desempenho foi classificado de acordo com os critérios apresentados em Sousa et al. (2010) e o nível de significância do coeficiente de correlação foi avaliado pelo teste t-Student.

\section{RESULTADOS E DISCUSSÃO}

A variação diária da evapotranspiração do coqueiro ( $\left.\mathrm{ET}_{\mathrm{c}}\right)$, a partir do lisímetro de grande porte, da evapotranspiração de referência $\left(E T_{o}\right)$ e os totais mensais de precipitação são apresentados na Figura 1. O lisímetro utilizado neste estudo, que realiza o balanço hídrico no solo completo através do cômputo das entradas (precipitação e irrigação) e saídas de água (evapotranspiração e drenagem) durante o período experimental, foi suficientemente preciso para detectar as variações de massa no interior do equipamento.
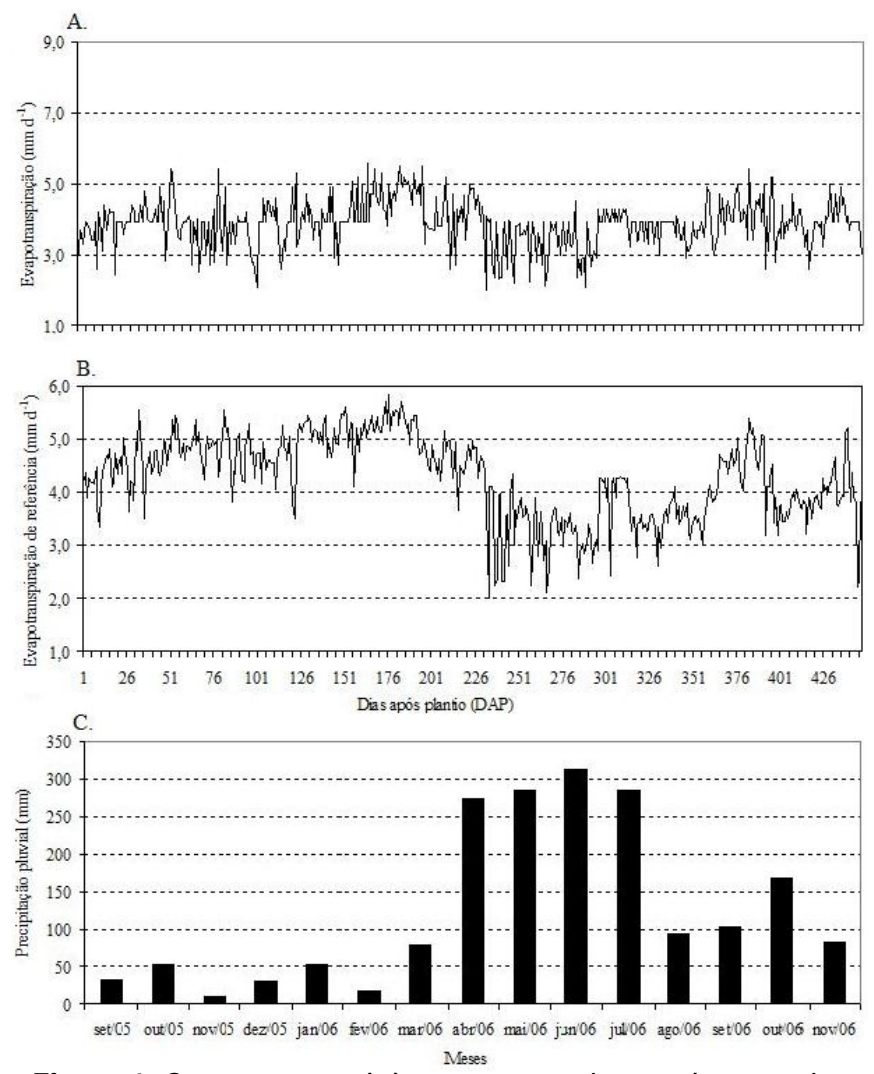

Figura 1. Curso temporal da evapotranspiração do coqueiro anão-verde irrigado (A), evapotranspiração de referência (B) e os totais mensais da precipitação pluvial durante 0 período experimental (C)

Os dados inconsistentes de $\mathrm{ET}_{\mathrm{c}}$ são obtidos, em geral, em lisímetros devido a vários problemas, sendo a principal fonte de erros a pressão que o vento exerce sobre sua superfície (López-Urrea et al., 2006). Neste particular, tal fonte de erros possivelmente não tenha interferido, de forma determinante, nas medições da $\mathrm{ET}_{\mathrm{c}}$ da fase de crescimento do coqueiro anãoverde, face à baixa velocidade média do vento registrada na região de estudo, que foi de apenas $1,6 \mathrm{~m} \mathrm{~s}^{-1}$. Ainda segundo esses autores, outras fontes de erro que levam à eliminação de alguns dias de medições com lisímetros são a falta de calibração 
da célula de carga, a ruptura do fio terra e as falhas no data logger; apesar disto, durante o período experimental desta pesquisa todos esses detalhes foram cuidadosamente observados para que o equipamento oferecesse medições precisas da $\mathrm{ET}_{\mathrm{c}}$ do coqueiro. A média diária e o desvio padrão da evapotranspiração do coqueiro anão-verde durante os 15 meses do período experimental foram de $3,9 \pm 0,7 \mathrm{~mm}$, com valores máximo e mínimo de 5,6 e 2,0 mm, respectivamente (Figura 1A).

A evapotranspiração do coqueiro acumulada durante o período experimental foi de $1.727,3 \mathrm{~mm}$, enquanto na fase de crescimento da planta, que correspondeu ao período de 1 de janeiro a 23 de novembro de 2006, a ET $_{\mathrm{c}}$ acumulada foi 1.263,3 $\mathrm{mm}$. A diferença entre os valores da evapotranspiração do coqueiro obtidos no presente estudo com aqueles apresentados por Azevedo et al. (2006), está associada à idade da planta, além das diferenças entre as condições de clima e solo onde foram realizadas as duas pesquisas. Em estudos sobre a evapotran spiração do coqueiro, também na região dos tabuleiros costeiros do Estado de Sergipe, Sousa et al. (2006) encontraram valores do consumo hídrico por ano dessa palmeira variando entre 870,8 a $1.090,6 \mathrm{~mm}$, para os tratamentos de irrigação variando de $50 \mathrm{~L}_{\text {planta }}^{-1} \mathrm{~d}^{-1}$ a $150 \mathrm{~L}_{\text {planta }}{ }^{-1} \mathrm{~d}^{-1}$, respectivamente.

A variação diária da $\mathrm{ET}_{\mathrm{o}}$ durante o período experimental é exibida na Figura 1B. A média diária e o desvio padrão da $\mathrm{ET}_{\mathrm{o}}$ foram 4,2 $\pm 0,8 \mathrm{~mm}$, com máximos e mínimos de 5,8 e 2,0, respectivamente. Como a ET representa a demanda potencial da atmosfera, caracterizada pelo poder evaporativo do ar, os menores valores dessa variável ocorreram justamente no período chuvoso, ou seja, entre os meses de abril e junho (Figura 2). Os períodos com valores máximos e mínimos de $\mathrm{ET}_{\text {o }}$ coincidem com os extremos da $\mathrm{ET}_{\mathrm{c}}$ (Figura 1A) nos mesmos períodos. Por outro lado, a $\mathrm{ET}_{\mathrm{o}}$ acumulada no período de 1 de setembro de 2005 a 21 de novembro de 2006 foi $1.912,5 \mathrm{~mm}$, enquanto apenas no ano de 2006 ela foi de 1.349,1 mm. Portanto, a demanda atmosférica foi $9,7 \%$ maior que o consumo hídrico do coqueiro durante todo o período experimental; já durante sua fase de crescimento a demanda atmosférica foi $6,3 \%$ maior do que o consumo hídrico dessa palmeira.

O período com valores mínimos de ET compreende à estação chuvosa da região de estudo (Figura 1C), que apresentou o total máximo de chuvas de $313,1 \mathrm{~mm}$ no mês de junho; inversamente, o valor máximo da $\mathrm{ET}_{\mathrm{c}}$ do coqueiro ocorreu no período seco da área de estudo, que compreende os meses de setembro a fevereiro, cujo resultado sugere que os dados diários de evapotranspiração do coqueiro, obtidos por lisimetria, são fortemente influenciados pelo total de água que atinge a área de captação do equipamento, em face da sua sensibilidade à drenagem de água no perfil do solo. Utilizando o método do balanço hídrico no solo, na região do Platô de Neópoles, também no Estado de Sergipe, Azevedo et al. (2006) obtiveram valores da evapotranspiração do coqueiro anãoverde, com seis anos de idade, variando entre 2,5 e $3,2 \mathrm{~mm} \mathrm{~d}^{-1}$.

A Figura 2 exibe o curso temporal do coeficiente de cultura $\left(\mathrm{K}_{\mathrm{c}}\right)$ do coqueiro anão-verde irrigado durante o período de 1 de setembro de 2005 a 21 de novembro de 2006. A média do $\mathrm{K}_{\mathrm{c}}$ do coqueiro durante este período foi 0,9 , com valores mínimo e máximo de 0,3 e 1,3, respectivamente. Os valores extremos do coeficiente de cultura aparecem, respectivamente, nos períodos de mínima e máxima evapotranspiração do coqueiro. Miranda et al. (2007) determinaram a evapotranspiração pelo método do balanço hídrico no solo e o coeficiente de cultura do coqueiro anão-verde no período de 11 a 41 meses após o plantio na região litorânea do Estado do Ceará; os autores observaram que a evapotranspiração do coqueiro variou de 0,51 a $5,01 \mathrm{~mm}$ $\mathrm{d}^{-1}$ e o coeficiente de cultura de 0,63 a 1,0, com média, durante todo o período analisado, de 1,02.

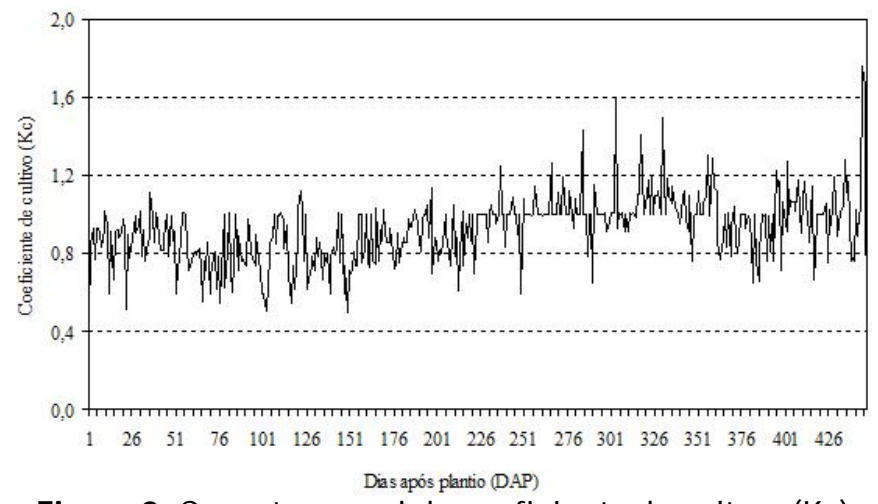

Figura 2. Curso temporal do coeficiente de cultura $(\mathrm{Kc})$ da fase de crescimento do coqueiro anão-verde durante 0 período experimental (setembro/2005 - novembro/2006)

O maior valor do $\mathrm{K}_{\mathrm{c}}$ do coqueiro ocorreu durante a fase de crescimento vegetativo, precisamente aos 230 dias após plantio. Por outro lado, os menores valores do $K_{c}$ do coqueiro foram obtidos nos primeiros dias após plantio, caracterizados pela fase de menor demanda hídrica dessa palmeira cultivada nas condições edafoclimáticas de realização desta pesquisa. Apesar do estudo não contemplar as fases de florescimento e desenvolvimento dos frutos do coqueiro, os resultados apresentados neste trabalho sobre a evapotranspiração e o consumo hídrico são úteis no planejamento e manejo eficiente da irrigação desta palmeira nas suas fases fenológicas iniciais, que são as mais importantes no cultivo de qualquer cultura, além de pouco estudadas.

O valor médio do $\mathrm{K}_{\mathrm{c}}$ obtido no presente estudo $\left(\mathrm{K}_{\mathrm{c}}=0,9\right)$, é semelhante àquele recomendado para palmeiras, por Allen et al. (1998), de 1,0. O curso temporal do coeficiente de cultura do coqueiro apresentado na Figura 2 é bastante variável ao longo do período de observação; entretanto, a fase inicial da palmeira (100 primeiros dias após plantio) é caracterizada por apresentar baixos valores do $\mathrm{K}_{\mathrm{c}}$ e, em seguida, ocorre um crescimento acentuado da curva do $\mathrm{K}_{\mathrm{c}}$, logo após, se estabilizar até o final da fase de crescimento vegetativo.

A relação entre a evapotranspiração do coqueiro obtida por lisimetria e pelo método da razão de Bowen, é exibida na Figura 3 , com coeficiente de determinação $\left(\mathrm{R}^{2}\right)$ estatisticamente significativo a nível de $1 \%$ de probabilidade, pelo teste tStudent. As eventuais diferenças acentuadas entre os valores medidos e observados de evapotranspiração podem estar associadas a problemas operacionais do equipamento durante a estação chuvosa e em dias secos com rajadas de vento (Pereira et al., 2002) ou à ausência de "fetch" suficiente para a aplicação 
do método da razão de Bowen na obtenção dos componentes do balanço de energia (Silva et al., 2007).

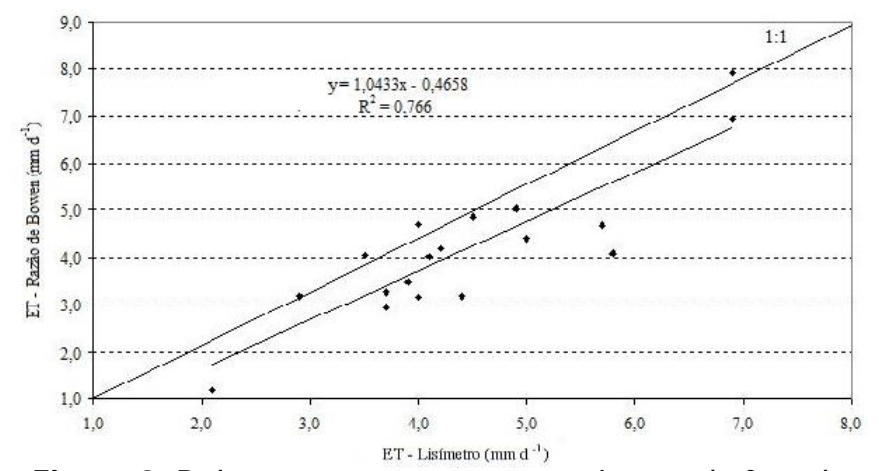

Figura 3. Relação entre a evapotranspiração da fase de crescimento do coqueiro anão-verde obtida por lisimetria (ET - Lisímetro) e pelo método da razão de Bowen (ET Razão de Bow en)

A comparação entre os valores da evapotranspiração obtidos por medições lisímetricas e através do balanço de energia baseado na razão de Bowen, foi realizada através de análise de regressão linear, erro quadrático médio e índice de concordância. As medições lisimétricas foram assumidas como variáveis dependentes e os dados da razão de Bowen, como variáveis independentes. Os dados da evapotranspiração por lisimetria superestimam aqueles obtidos pelo método do balanço de energia baseado na razão de Bowen em 61\%, com erro quadrático médio (EQM) de $0,71 \mathrm{~mm} \mathrm{~d}^{-1}$. López-Urrea et al. (2006), comparando os valores da evapotranspiração de referência por sete diferentes métodos com medições lisímetricas, encontraram valores de EQM variando de 0,67 a $1,58 \mathrm{~mm} \mathrm{~d}^{-1}$, sendo o menor erro associado ao melhor ajuste, que foi com a equação de Penman-Monteith. O lisímetro, que foi construído, instalado e calibrado conforme descrito na Parte I deste trabalho, apresentou boas condições de funcionamento que permitiram a obtenção de medidas de evapotranspiração em concordância com as estimativas realizadas pelo método do balanço de energia baseado na razão de Bowen na região estudada.

O índice de concordância (d) entre os valores da evapotranspiração obtidos por medições lisimétricas e através do balanço de energia baseado na razão de Bowen, foi de 0,92 , enquanto o índice de desempenho $(c=r . d)$ foi de 0,81 , classificado como muito bom, segundo os critérios de avaliação deste índice, apresentados por Sousa et al. (2010). O índice de concordância é uma medida relativa das diferenças entre as variáveis; portanto, o valor de 0,92 para este índice também representa uma boa relação entre os valores medidos e estimados da evapotranspiração da fase de crescimento do coqueiro, pois a perfeita concordância ocorre quando $d=1$. Este resultado assegura que o método micrometeorológico da razão de Bowen pode ser usado com boa precisão na obtenção da evapotranspiração do coqueiro.

Resultados de estudos anteriores (Silva et al., 2005; Gavilán \& Berengena, 2007) também mostraram boa concordância entre os valores das medições lisimétricas com os dados do modelo de Bowen. Nesta linha de pesquisa, Loos et al. (2007) encontraram que os valores da evapotranspiração pelos métodos Penman, Penman-Monteith e de Haude superestimaram os dados obtidos por lisímetro de pesagem em Munique, Alemanha, quando simularam o balanço hídrico no solo com base nesse equipamento. Por outro lado, Liu et al. (2007) usaram lisímetro de pesagem para determinar a eficiência do uso de água em frutífera (morango) em Copenhaque, com resultados bastante satisfatórios. Este equipamento também foi utilizado por Tyagi et al. (2000) visando determinar a evapotranspiração e o coeficiente do arroz e do girassol cultivados na Índia. Os resultados obtidos nesta segunda parte do presente trabalho permitem observar que eles podem ser usados no planejamento e no manejo eficiente da irrigação do coqueiro em ambientes similares ao clima da região em que foi desenvolvida esta pesquisa.

\section{Conclusões}

1. O relacionamento entre os valores da evapotranspiração medidos por lisímetro e obtidos pelo modelo da razão de Bowen, é classificado como muito bom, sugerindo que este método pode ser usado com precisão na estimativa da evapotranspiração de plantas de grande porte.

2. O lisímetro de pesagem de grande porte mostrou-se capaz de responder às variações dos principais componentes do balanço hídrico do solo, que influenciam na evapotranspiração do coqueiro anão-verde.

\section{LITERATURA CITADA}

Allen, R. G.; Pereira, L. A.; Raes, D.; Smith, M. Crop evapotranspiration (guidelines for computing crop water requirements). Rome: FAO, 1998. 297p. Irrigation and Drainage Paper, 56

Azevedo, P. V. de; Silva, B. B. da.; Silva, V. de P. R. da. Water requirements of irrigated mango orchards in northeast Brazil. Agricultural Water Management, v.58, p.241-254, 2003.

Azevedo, P. V. de; Soares, J. M.; Silva, V. de. P. R. da; Silva, B. B. da; Nascimento, T. Evapotranspiration of "Superior" grapevines under intermittent irrigation. Agricultural Water Management, v.95, p.301-308, 2008.

Azevedo, P. V. de; Sousa, I. F. de; Silva, B. B. da; Silva, V. de. P. R. da. Water-use efficiency of dwarf-green coconut (Cocos nucifera L.) orchards in northeast Brazil. Agricultural Water Management, v.84, p.259-264, 2006.

Borges, C. J. R.; Silva, V de. P. R. da; Campos, J. H. B. da C.; Azevedo, P. V. de; Moura, M. S. B.; Soares, J. M.; Silva, B. B da. Influência do calor armazenado no sistema solo-planta no balanço de energia em pomar de mangueiras. Revista Brasileira de Engenharia Agrícola e Ambiental, v.12, p.393399, 2008.

Gavilán, P.; Berengena, J. Accuracy of the Bowen ratio-energy balance method for measuring latent heat flux in a semiarid advective environment. Irrigation Science, v.25, p.127-140, 2007. 
Lins, P. M. P.; Farias Neto, J. T.; Muller, A. A. Avaliação de híbridos de coqueiro (Cocos nucifera $\mathrm{L}$.) para a produção de frutos e de albúmem sólido fresco. Revista Brasileira de Fruticultura, v.25, p.468-470, 2003.

Liu, F.; Savic', S.; Jensen, C. R.; Shahnazari, A.; Jacobsen, S. E.; Stikic', R.; Andersen, M. N. Water relations and yield of lysimeter-grown strawberries under limited irrigation. Scientia Horticulturae, v.111, p.128-132, 2007.

Loos, C.; Gayler, S.; Priesack, E. Assessment of water balance simulations for large-scale weighing lysimeters. Journal of Hydrology, v.335, p.259-270, 2007.

Lopes-Urrea, R.; Olalla, F. M. S.; Fabeiro, C.; Moratalla, A. Testing evapotranspiration equations using lysimeter observations in a semiarid climate. Agricultural Water Management, v.85, p.15-26, 2006.

Miranda, F. R.; Gomes, A. R. M.; Oliveira, C. H. C.; Montenegro, A. A. T.; Bezerra, F. M. L. Evapotranspiração e coeficientes de cultivo do coqueiro anão-verde na região litorânea do Ceará. Revista Ciência Agronômica, v.38, p.129-135, 2007.

Pereira, A. R.; Santiago, A. S.; Maggioto, S. R.; Folegatti, M. V. Problemas operacionais com lisímetro de pesagem durante a estação chuvosa e em dias secos com rajadas de vento. Revista Brasileira de Agrometeorologia, v.10, p.51-56, 2002.

Silva, L. D. B.; Folegatti, M. V.; Villa Nova, N. A. Evapotranspiração do capim tanzânia obtida pelo método de razão de Bowen e lisímetro de pesagem. Engenharia Agrícola, v.25, p.705-712, 2005.

Silva, V. de. P. R. da; Azevedo, P. V. de; Silva, B. B. da. Surface energy fluxes and evapotranspiration of a mango orchard grown in semiarid environment. Agronomy Journal, v.99, p.1391-1396, 2007.

Silva, V. de. P. R. da; Campos, J. H. B. da C.; Azevedo, P. V. de. Water-use efficiency and evapotranspiration of mango orchard grown in northeastern region of Brazil. Scientia Horticulturae, v.102, p.467-472, 2009.
Silva, V. de. P. R. da; Belo Filho, A. F.; Silva, B. B. da; Campos, J. H. B. da C. Desenvolvimento de um sistema de estimativa da evapotranspiração de referência. Revista Brasileira de Engenharia Agrícola e Ambiental, v.9, p.547-553, 2005.

Sousa, I. F. de; Silva, V. de. P. R. da; Sabino, F. B.; Aguiar Neto, A. O.; Silva, B. K. N.; Azevedo, P. V. de. Evapotranspiração de referência nos perímetro irrigados do Estado de Sergipe. Revista Brasileira de Engenharia Agrícola e Ambiental, v.14, p.633-644, 2010.

Sousa, I. F. de; Silva, V. de. P. R. da; Silva, B. B. da; Cintra, F. L. D. Estimativa da evapotranspiração do coqueiro irrigado pelo método do balanço hídrico no solo. Revista Brasileira de Agrometeorologia, v.14, p.1-10, 2006.

Souza, C. B.; Silva, B. B. da; Azevedo, P. V. de; Silva, V. de. P. R. da. Fluxos de energia e desenvolvimento da cultura do abacaxizeiro. Revista Brasileira de Engenharia Agrícola e Ambiental, v.12, p.400-407, 2008.

Tyagi, N. K.; Sharma, D. K.; Luthra, S. K. Determination of evapotranspiration and crop coefficients of rice and sunflower with lysimeter. Agricultural Water Management, v.45, p.41-54, 2000.

Unland, H. E.; Houser, P. R.; Shuttleworth, W. J.; Yang, Z. L. Surface flux measurements and modelling at a semi-arid sonoran desert site. Agricultural and Forest Meteorology, v.82, p.119-153, 1996.

Vaughan, P. J.; Trout, T. J.; Ayars, J. E. A processing method for weighing lysimeter data and comparison to micrometeorological ETo predictions. Agricultural Water Management, v.88, p.141-146, 2007.

Willmott, C. J. Some comments on the evaluation of model performance. Bulletin of the American Meteorological Society, v.63, p.1309-1313, 1982. 